\title{
Une innovation-produit au service de la stratégie d'un territoire touristique transfrontalier émergent
}

Le cas de la randonnée franco-belge « Rando Famili »

Using product innovation to develop an emerging crossborder tourist

destination. The case of Franco-Belgian hiking: Rando Famili

Fabrice Caudron et Céline Thomas

\section{OpenEdition}

\section{Journals}

Édition électronique

URL : http://journals.openedition.org/tourisme/1396

DOI : 10.4000/tourisme.1396

ISSN : 2492-7503

Éditeur

Éditions touristiques européennes

Référence électronique

Fabrice Caudron et Céline Thomas, « Une innovation-produit au service de la stratégie d'un territoire touristique transfrontalier émergent », Mondes du Tourisme [En ligne], 13 | 2017, mis en ligne le 30 décembre 2017, consulté le 20 mars 2020. URL : http://journals.openedition.org/tourisme/1396 DOI : https://doi.org/10.4000/tourisme.1396

Ce document a été généré automatiquement le 20 mars 2020.

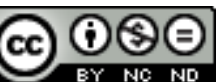

Mondes du tourisme est mis à disposition selon les termes de la licence Creative Commons Attribution - Pas d'Utilisation Commerciale - Pas de Modification 4.0 International. 


\section{Une innovation-produit au service de la stratégie d'un territoire touristique transfrontalier émergent}

Le cas de la randonnée franco-belge « Rando Famili »

Using product innovation to develop an emerging crossborder tourist

destination. The case of Franco-Belgian hiking: Rando Famili

Fabrice Caudron et Céline Thomas

\section{Introduction}

1 Le présent article traite d'une innovation-produit sur un territoire touristique transfrontalier. Le programme Interreg IV de l'Union européenne a soutenu l'émergence d'un projet franco-belge de développement de la randonnée : «Inno Rando Pour Tous ». Ce projet a permis la mise en place d'une coopération transfrontalière avec la création de nouveaux produits touristiques et l'emploi d'une nouvelle application mobile. À travers cette collaboration, les territoires concernés ont créé un nouveau produit à destination d'un public familial : «Rando Famili ». La problématique de notre cas d'étude porte ainsi sur les conditions d'émergence d'une innovation dans le domaine du tourisme.

2 Plusieurs types de questionnements émerge de cette coopération entre acteurs transfrontaliers, portant à la fois sur la question de la segmentation clients, sur la nature de l'expérience de consommation et sur la gouvernance transfrontalière (autour de la construction d'un nouveau territoire touristique).

3 Nous présenterons dans un premier temps le contexte et le champ théorique de cet article. Puis, nous présenterons le projet «Rando Famili» avant de proposer une discussion sur les implications managériales d'un tel projet. 


\section{Contexte et champs théorique : territoire touristique transfrontalier et innovation-produit}

\subsection{Un territoire touristique en construction}

4 Les frontières nationales servent le plus souvent à définir un territoire touristique pour en déterminer la fréquentation mais également, plus largement, l'ensemble des indicateurs permettant de caractériser l'importance du tourisme dans l'économie d'un pays. Les partenariats transfrontaliers constituent donc une pratique différente par rapport à ce que les acteurs du tourisme font habituellement dans le cadre de leur activité. Ces partenariats institutionnels posent de nombreuses questions quant au leadership (et plus largement à la souveraineté) lié au développement d'un projet commun (Timothy, 1999). Les mêmes travaux insistent sur l'importance des traités bilatéraux, dans le contexte nord-américain, pour établir des règles de gouvernance claires. Au sein de l'Union européenne, le cadre est déterminé par l'existence de programmes de grande envergure. Le programme Interreg constitue notamment l'une de initiatives communautaires les plus importantes, du moins celle qui a permis la mise en œuvre du plus grand nombre de projets à l'échelle européenne (Bolgherini et Roux, 2008).

5 Sur la zone géographique concernée par le projet « Inno Rando Pour Tous », l'enjeu est explicite: "le programme France-Wallonie-Vlaanderen: régions françaises et belges qui coopèrent pour effacer la frontière! Le programme de coopération territoriale européen Interreg France-Wallonie-Vlaanderen s'inscrit dans une volonté de favoriser les échanges économiques et sociaux entre cinq régions frontalières: le Nord-Pas de Calais, la Champagne-Ardenne et la Picardie, en France; la Wallonie et la Flandre, en Belgique. Il vise à associer des compétences communes tout en valorisant les richesses de chaque région concernée, et ce, au bénéfice des populations de la zone.» (extrait du site internet Interreg France - Wallonie Vlaanderen : http://interreg5.interreg-fwvl.eu/)

6 La politique européenne promeut ici explicitement la constitution d'un nouvel espace touristique (Denert et Hurel, 2000). Au-delà de la création d'un nouveau territoire, voire d'une nouvelle destination, ce type de projet transfrontalier vise à favoriser l'échange et le transfert de ressources et de savoirs en vue de la constitution de nouveaux avantages compétitifs. Les trois facteurs d'attractivité majeurs d'un territoire transfrontalier sont sa localisation géographique (d'où peut découler un intérêt naturel ou culturel), sa dimension géopolitique (histoire, héritage, etc.) et son type de développement touristique (Weidenfeld, 2013).

7 Le projet franco-belge "Inno Rando Pour Tous" couvre un vaste territoire transfrontalier de randonnée au nord de Paris et sur une partie de la Belgique. Il associe, pour le versant français, les agences de développement et de réservation touristiques (encore dénommées comités départementaux du tourisme au lancement du projet) et les conseils généraux du Nord et de l'Aisne, et, pour le versant belge, l'Intercommunale IDETA, également Maison du tourisme de la Wallonie picarde. Il a été financé dans le cadre du programme Interreg IV France-Wallonie-Vlaanderen 2007-2013, sous le titre « Inno Rando Pour Tous ». Le budget global de l'opération est de 3945000 euros. L'objectif opérationnel du partenariat vise à utiliser au mieux le potentiel des technologies émergentes web et mobiles afin de satisfaire les besoins en 
randonnée d'un segment de clientèle particulier: le public familial (adultes avec enfants jusqu'à 12 ans). Dans ce cadre, ce sont essentiellement les actions portant sur l'innovation-produit «Rando Famili » qui nous intéressent. La démarche stratégique des acteurs rejoint l'objectif principal assigné au marketing territorial : identifier les besoins amont pour en déduire des actions, dans le cadre des frontières d'un territoire défini (Debabi et Daouas, 2015). Ce diagnostic amont a amené les partenaires à lancer une innovation dans le domaine du tourisme de randonnée afin de constituer un nouveau facteur d'attractivité du territoire.

8 La particularité de notre cas d'étude tient à la pluralité des acteurs mobilisés. Nous sommes en effet à la charnière d'un territoire transfrontalier qui réunit des Français et des Belges; plus précisément des Français, des Belges néerlandophones et des Belges francophones.

\subsection{Champ théorique : une innovation-produit dans le domaine de la randonnée touristique}

9 Au sens de l'Insee, l'innovation de produit est l'«introduction sur le marché d'un produit (bien ou service) nouveau ou significativement amélioré au regard de ses caractéristiques essentielles.» Appliqué au domaine touristique, le concept d'innovation se révèle néanmoins plus complexe, dans la mesure où « l'innovation dans le secteur du tourisme provient généralement d'éléments issus de problématiques environnementales, culturelles ou encore sociales » (Leroux, 2015). L'innovation dans le secteur touristique mobilise ainsi une hétérogénéité d'acteurs ayant souvent recours aux technologies de l'information et de la communication (TIC).

10 Le secteur du tourisme a mis du temps à s'approprier les innovations issues des TIC mais celles-ci constituent désormais un axe de développement stratégique majeur pour le secteur (Gallouj et Leroux, 2011). Nous analysons ici le cas d'une innovation technique, qui correspond à l'apparition d'un nouveau service pour le consommateur. Ce nouveau service permet d'accéder à un parcours de randonnée amélioré par l'utilisation d'une application mobile. On se trouve ici dans une démarche de M-tourism qui relève de la « relation de détermination » largement conditionnée par la demande de service et de qualité émise par le touriste (Gallouj et Leroux, 2011).

11 La randonnée constitue un champ particulier du tourisme, une forme de tourisme de niche. À ce titre, elle peut être un lieu privilégié d'innovation, dans le sens où elle se doit d'adopter une approche très pragmatique, proche des « motivations des touristes » (Carlisle et al., 2013).

12 L'offre de randonnée constitue par ailleurs un élément important du développement d'un tourisme durable de qualité en milieu rural, comme c'est par exemple le cas sur le territoire Picard (François, 2004 ; Leroux, 2015). C'est également un moteur important du développement d'offres de services pourvoyeuses d'emplois et de retombées pour le territoire, même si la mesure précise des impacts s'avère difficile (Marsac et al., 2012) : restaurants de terroir, cafés-rando, hébergements ruraux de toutes sortes, guides nature, etc. Bien que constituant la première activité de pleine nature pratiquée en France et l'activité physique préférée des Français en vacances (d'après l'étude Statistica 2016, Classement des activités sportives préférées des français en novembre 20161), le marketing de la randonnée a rarement dépassé une segmentation " fonctionnelle » en lien avec la nature de la pratique (grande randonnée, boucles à la journée, boucles à la 
demi- journée) ou le terrain et l'environnement du circuit lui-même, directement lié à l'image de la destination (montagne/campagne/lande, etc.).

D'un point de vue global, la randonnée ne permet pas de retombées économiques directes et immédiates (les sentiers sont balisés et accessibles à tous, sans «ticket d'entrée » la plupart du temps). Le développement d'une offre spécifique repose donc d'abord sur des acteurs non marchands, à savoir, selon les territoires, le secteur public et/ou associatif. Son financement reste majoritairement le fait d'acteurs publics qui n'en tirent aucun profit direct, en dehors de la vente de cartes ou de topoguides. Ces particularités amènent certains acteurs du secteur à imaginer des solutions innovantes pour des publics hétérogènes.

Saayman et Viljoen (2016) ont élaboré une typologie des randonneurs, répartis en trois catégories principales et aux motivations très différentes :

- les «admirateurs", qui apprécient la proximité avec la nature, la beauté des paysages et l'isolement par rapport au contexte urbain ;

- les « aventuriers ", qui sont largement attirés par la dimension physique de la randonnée en tant que performance sportive, autonome et parfois risquée ;

- les « amateurs", qui regroupe un public familial, désireux d'apprendre sur la nature et qui privilégie l'aspect relationnel; c'est cette catégorie de randonneurs qui constitue la cible principale du projet « Inno Rando Pour tous ».

\section{Le cas d'une randonnée franco-belge}

15 Notre étude de cas se situe au carrefour de l'innovation-produit et du tourisme de randonnée, dans le contexte d'un territoire touristique transfrontalier émergent. Cette expérience rejoint le développement d'offres qualifiées pour un public familial dans le cadre du tourisme durable (Salvador-Perignon, 2012; Siau, 2007; Courvoisier et Courvoisier, 2005): produits artisanaux, musées, attractions, hébergements, loisirs sportifs de nature, etc.

\subsection{Méthodologie}

«Rando Famili» constitue l'un des axes de déploiement du projet «Inno Rando Pour Tous ", à côté du déploiement d'itinéraires de randonnée pour personnes à mobilité réduite, et s'inscrit lui-même dans le cadre du programme Interreg IV France-WallonieVlaanderen 2007-2013. Ce projet a fait l'objet d'une convention qui couvre la période s'étalant du $1^{\mathrm{er}}$ octobre 2008 au 31 mars 2015. Notre étude a consisté à extraire du projet global l'ensemble des éléments d'information portant sur l'innovation-produit «Rando Famili ». Elle repose sur quatre types de données:

- le rapport d'activités final du projet «Inno Rando Pour Tous », au 31 décembre 2014 (qui contient l'ensemble des éléments relatifs à la conduite du projet),

- les supports de communication externe du projet, dont le site web : www.randofamili.com/ pour la version francophone et www.familiewandelingen.com pour la version néerlandophone,

- les comptes-rendus des réunions de pilotage du projet,

- des extraits d'échanges sur la plateforme collaborative du projet et des échanges informels avec l'opérateur chef de file du projet, pour la partie wallonne. 
17 L'étude de cas a été corédigée avec l'un des acteurs du projet, qui en était le gestionnaire principal pour la partie belge francophone.

\subsection{Présentation de l'étude de cas}

\section{Le projet et sa gouvernance}

Jusqu'à la création de ce projet, la randonnée bénéficiait sur ces territoires d'un marketing s'adressant à une clientèle adulte, individus ou groupes. Les partenaires ont choisi de cibler le public familial en sélectionnant des circuits à haute valeur ajoutée.

Trois actions stratégiques ont ainsi été menées: les deux premières (actions 1 et 2) visaient à développer l'accessibilité de l'offre au public PMR (personnes à mobilité réduite), la dernière (action 3 ) concerne l'innovation-produit et la politique de communication correspondante. C'est essentiellement sur l'action 3 que s'est porté notre intérêt, avec la production de «contenus riches et innovants". Nous ne mentionnons ici que les actions communes directement liées au projet, excluant par làmême la communication spécifique mise en œuvre par chacun des partenaires, au travers notamment des réseaux sociaux.

Encadré 1. Synthèse des actions réalisées dans le cadre du projet « Rando Famili "

Action 1 : aménagement des itinéraires PMR (personnes à mobilité réduite)

Action 2 : développement et structuration de l'offre PMR (équipement)

Action 3 : innovation, promotion

Réalisation de :

- un site internet transfrontalier,

- des contenus riches (23 vidéos, 263 capsules audios, une soixantaine de topo-

fiches),

- une interface mobile (application « Rando Famili » pour IOS et Androïd),

- une campagne promotionnelle de webmarketing (espace publicitaire Google,

Facebook et sites affinitaires, du type Pays du Nord),

- des publications en français et en néerlandais,

- la création d'une marque (« Rando Famili »).

Source : Rapport d'activités final du projet « Inno Rando Pour Tous », programme Interreg IV.

\section{Création de la marque « Rando Famili » et positionnement}

Dans le cadre de l'action 3, les partenaires ont donc créé une marque commune: « Rando Famili, des balades fun et facile à faire en famille ». Ce choix a été le fruit d'un processus de discussion visant à permettre l'émergence d'une coopération génératrice de valeur ajoutée pour chacun des acteurs. Ainsi, on voit se construire, petit à petit, au cours des séances de travail et sur la plateforme collaborative en ligne, un projet commun autour du public familial. Le résultat de ce processus apparait explicitement dans le rapport final du projet (p. 12) :

« $\mathrm{Au}$ cours de ces séances de travail, il est apparu que la clientèle familiale constituait une cible de clientèle partagée et essentielle à l'offre de randonnée sur les trois territoires associés. C'est aussi une clientèle qui est en demande de 
réassurance sur la pratique de la randonnée, car la présence d'enfants amène des contraintes (difficulté limitée des itinéraires, distances courtes, motivation de l'enfant à poursuivre jusqu'au bout). Les partenaires ont donc convenu de créer un dispositif innovant en adressant cette clientèle (sans précédent d'après les recherches effectuées) et la clientèle en situation de handicap. Chacun a convenu d'effectuer une sélection d'itinéraires pédestres (maximum 30 par territoire) convenant à la clientèle familiale sur la base de critères définis en commun (distance, difficulté, intérêt et services) et de les promouvoir sur un dispositif partagé, dénommé Rando Famili / Familie Wandelingen. "

\section{Les circuits « Rando Famili »}

21 Cette nouvelle «marque » regroupe 81 randonnées pédestres qualifiées pour le public familial (à travers des critères de distance, d'accessibilité et d'intérêt). Ces circuits ont vu leur valeur renforcée par la création de pastilles audios à écouter en chemin, d'une topo-fiche conviviale et attractive pour les enfants et de vidéos de séduction thématiques aidant au choix en amont. Les tracés et leurs contenus multimédias sont accessibles depuis l'application « Rando Famili » téléchargeable sur l'App Store et sur Google Play. À chaque fois des topo-fiches «familiales » ont été éditées : l'objectif était de générer de la valeur ajoutée pour le public cible en proposant des outils ludiques et conviviaux. C'est ainsi qu'une cinquantaine d'aquarelles ont été dessinées afin de représenter chaque itinéraire de façon moins technique qu'une carte-guide classique. Les 263 pastilles audios renforcent en outre la possibilité d'interprétation du circuit par le public en lui apportant des anecdotes ou des explications en lien avec les monuments ou les paysages rencontrés.

Parallèlement, 23 vidéos thématiques ont été réalisées. Elles sont accessibles à partir du site internet du projet, de sa chaîne YouTube (https://www.youtube.com/user/ familirando) ou de l'application mobile. La vidéo la plus regardée porte sur « Les bières de Wallonie picarde », avec plus de 8000 vues en début d'année 2015.

Figure 1. Exemple de topo-fiche familiale (extrait)

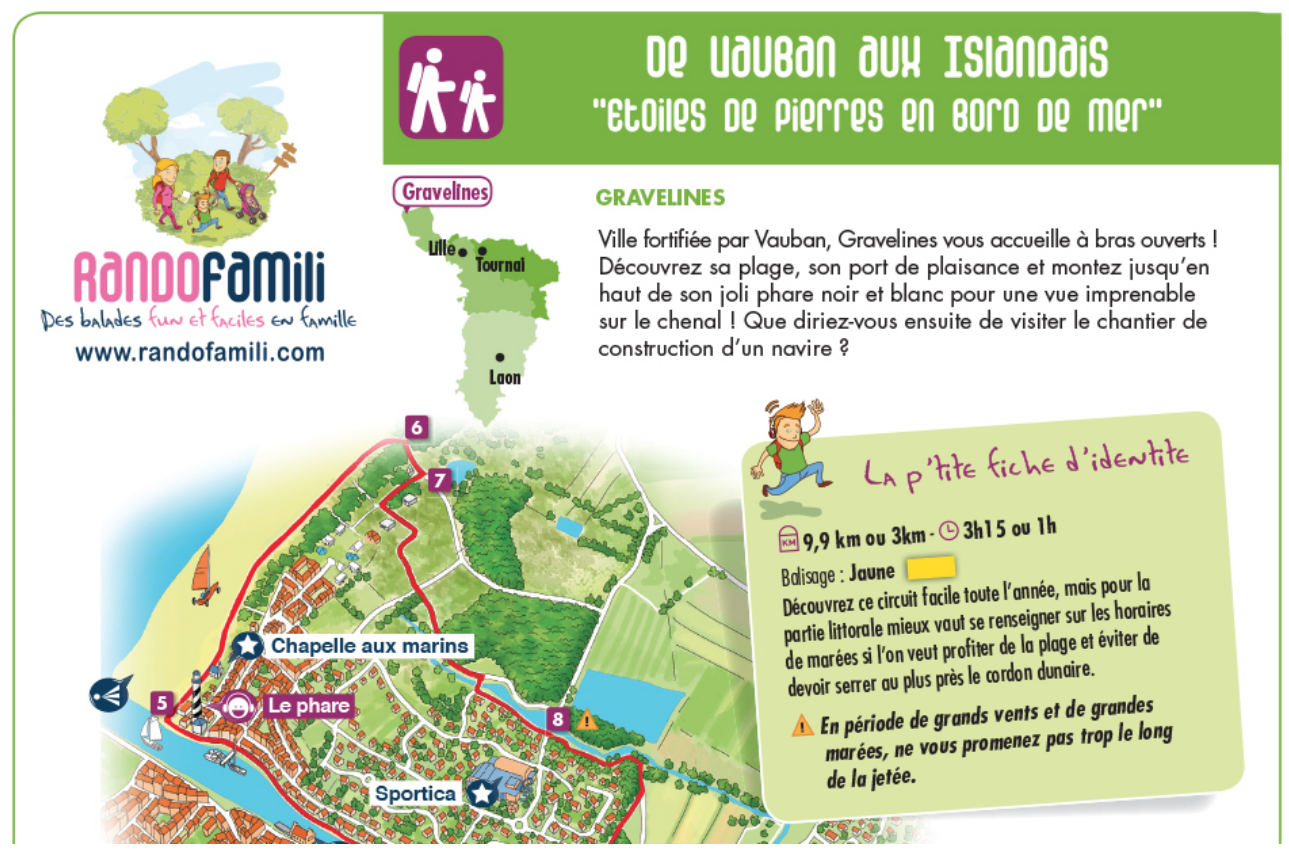




\section{Un site internet conçu selon une nouvelle segmentation clients}

\section{français et www.familiewandelingen.com en néerlandais (cible: clientèle belge} flamande). Sa création a été pilotée par un partenaire français du projet. Il a généré 98000 visites en 2013 et a été appuyé par une campagne de webmarketing qui s'est étalée sur 2012-2013. Le site propose une segmentation des randonnées (appelées «balades ») adaptées au public cible : par zone géographique, en fonction de leur durée et de la facilité d'accès (il existe par exemple un champ " accessible aux poussettes »). Plusieurs modalités de recherche complètent cette segmentation afin d'offrir au public une facilité de navigation.

Figure 2. La page d'accueil du site www.randofamili.com (version francophone)

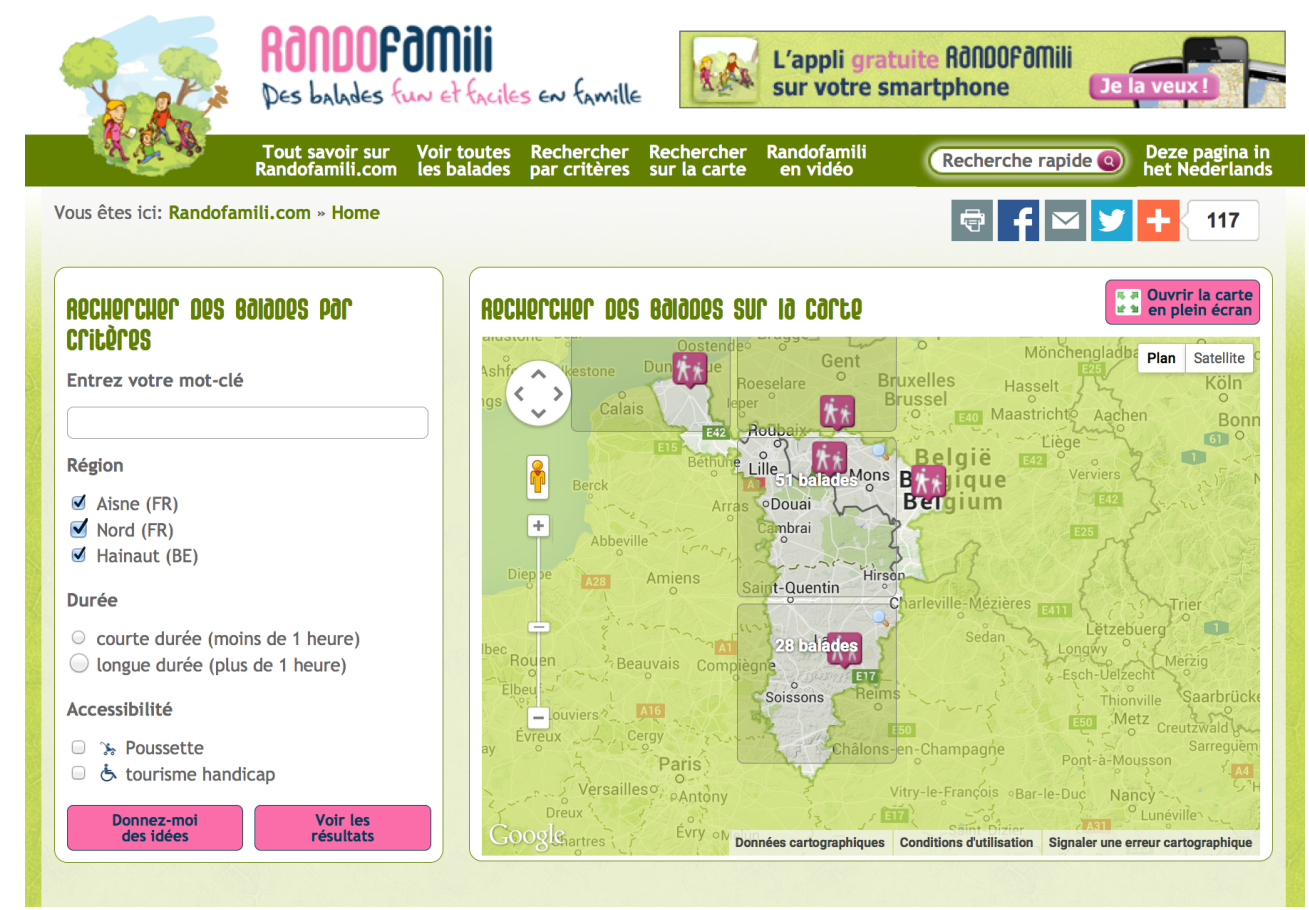

\section{Les applications mobiles}

C'est une des parties françaises du projet qui a piloté la création des applications destinées aux deux environnements les plus répandus: IOS et Android. Leurs fonctionnalités ont été conçues afin d'apporter une valeur ajoutée par rapport au site internet et aux topo-fiches. Les applications proposent donc les services suivants : la recherche d'un itinéraire « autour de moi » ou par critères, la visualisation légère d'un itinéraire (descriptif et photos), le téléchargement des contenus riches de l'itinéraire, le guidage GPS pendant la balade, le déclenchement automatique des pastilles audios et, enfin, le jeu Inspecteur rando pour les enfants.

Le résultat final ne semble pas complètement à la hauteur des espérances des partenaires, dans la mesure où la gestion de la diversité de la provenance des contenus (réunion des flux des systèmes d'information touristique de chaque partenaire) a rendu la réalisation de ces applications très complexe. Il apparaît, dans les comptes-rendus de 
réunions, que l'application est jugée " très lourde » et « compliquée d'utilisation » pour un « public amateur».

Figure 3. Capture d'écran IPhone de I'Appli « Rando Famili »

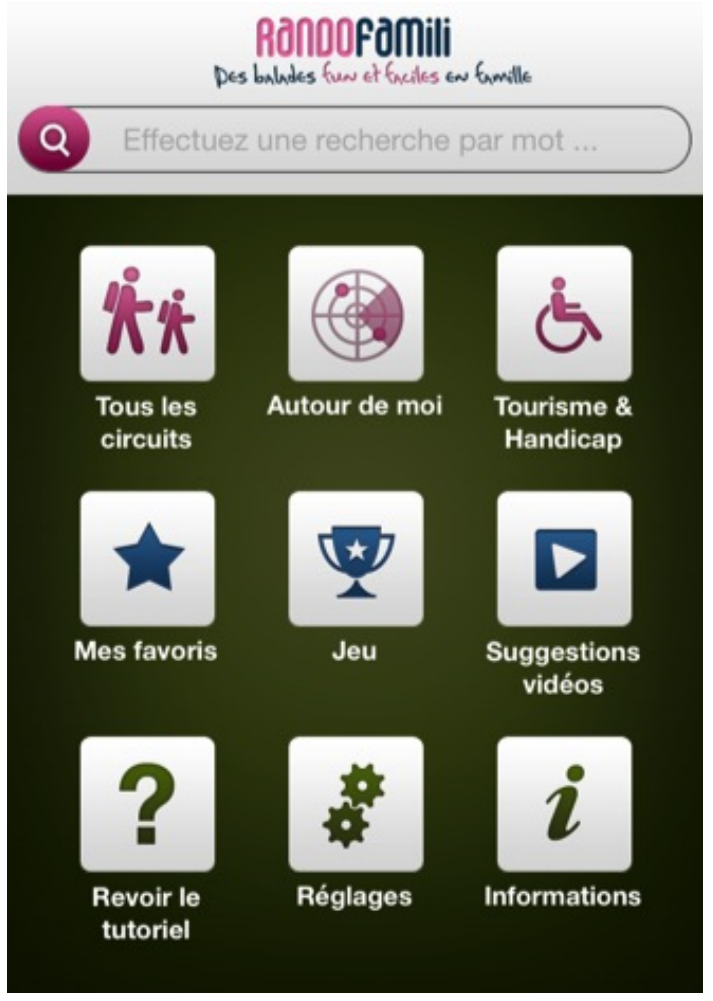

\section{Discussion}

L'étude de cas « Rando Famili » nous amène à mettre en valeur trois enseignements.

1) Les technologies émergentes web et mobiles permettent de penser des produits innovants pour de nouveaux segments de la clientèle touristique

Dans le cas présenté ici, l'innovation-produit est directement liée à l'émergence de nouveaux outils web et mobiles. Néanmoins, il ne s'agit pas uniquement d'une innovation instrumentale: les implications de l'utilisation des nouvelles technologies amènent à une redéfinition des segmentations "classiques" de la clientèle dans le secteur. Ainsi, l'application mobile et le site internet permettent-ils de proposer des usages adaptés à de nouveaux segments (Galloug et Leroux, 2011 ; Carlisle et al., 2013).

\section{2) L'expérience au cœur du tourisme de randonnée : un touriste actif}

L'expérience du randonneur peut être résumée en trois points: le paysage, le « moment» (les relations interpersonnelles notamment) et les émotions (Chhetri et al., 2004). La nature de cette expérience est intrinsèquement subjective. Dans la même étude, les mots utilisés par les randonneurs sont explicites: attracting, boring, challenging, crowding, enjoying, exciting, frustrating, motivating, stimulating, etc. La randonnée doit être mémorable pour devenir une expérience personnelle et émotionnelle à partir de laquelle le touriste pourra se mettre en scène (Saayman et Viljoen, 2016). 
31 Le point commun à toutes les analyses sur les expériences de consommation concerne l'importance des émotions ressenties (Ladwein, 2005). Apparaît par ailleurs l'importance de la dimension active du consommateur ; l'expérience de consommation joue un rôle central dans la satisfaction, la randonnée permet une mise en récit de soi à travers une expérience où le touriste est acteur d'une expérience renforcée par les nouveaux supports techniques qui sont mis à sa disposition (Filser, 2005).

3) Les implications d'une gouvernance transfrontalière dans la construction d'un nouveau territoire touristique

On retrouve ici l'enjeu de «similarité des structures » impliquées dans le projet afin de faciliter le processus d'innovation (Weidenfeld, 2013). Les proximités géographique, économique et managériale ont une influence majeure sur la réussite du processus d'innovation et le transfert de savoirs à l'échelle du nouveau territoire. Les acteurs impliqués dans le projet ont ainsi pour caractéristique de posséder des structures de promotion du tourisme assez similaires.

$\mathrm{Si}$ « la prise en compte de l'identité [d'un territoire] et l'engagement des élus sur la mise en cuvre de politiques touristiques durables sont au fondement de ce qui doit nécessairement devenir un projet partagé pour réussir » (Siau, 2007), les représentations portées par les acteurs peuvent évoluer tout au long du projet. L'originalité de la gouvernance d'un projet transfrontalier est d'encourager le processus d'innovation en transformant la représentation que les acteurs se font de leur propre territoire. À terme, le soutien des institutions européennes à ce type de projet permet l'émergence d'un nouveau territoire touristique, allant bien au-delà de ce que les élus envisageaient.

Ce processus de maturation peut permettre l'émergence d'une stratégie spécifique à la destination et, par voie de conséquence, la mise en place de produits touristiques innovants permettant un positionnement original du nouveau territoire (Maunier, 2007). À partir du moment où l'innovation-produit et l'association d'une diversité d'acteurs transforme la représentation que les acteurs touristiques eux-mêmes se font de leur territoire, en créant une nouvelle "personnalité » pour la destination, un «nouvel objet politique local» (Bartikowski et al., 2008 ; Haschar-Noé, 2009), c'est la stratégie de développement touristique elle-même qui est impactée. Dans le cas du projet «Rando Famili », les acteurs ont probablement pris conscience, à travers leur collaboration, du potentiel touristique que représente la fréquentation de chemins de randonnée par des pratiquants et pratiquantes dans un espace périurbain (Sahuc, 2010), ou du moins proche de grands centres urbains. Du côté français, la métropole lilloise comptait par exemple 1,1 million d'habitant en $2015^{2}$, alors que la partie la plus urbanisée du territoire transfrontalier en comptait 2,1 millions en $2008^{3}$.

Sur le plan opérationnel, un nouveau besoin émerge avec la nécessité d'une fonction de coordination des acteurs, en lien avec l'utilisation des outils numériques : la question se pose de la création d'un poste d'animateur numérique du territoire (Alexandre-Bourhis et al., 2013). Au-delà de la simple création d'un poste, une question plus large se pose : l'approche par filière/par produit, avec la constitution d'équipes spécifiques, amènerat-elle à la création de territoires touristiques sans identité propre? L'intrication des structures sera nécessairement croissante. 


\section{En guise de conclusion}

37 L'étude de cas présentée ici a fait l'objet d'un recueil de données qualitatives, essentiellement basé sur des ressources documentaires et des échanges informels confidentiels. Corédigée avec l'un des acteurs majeurs du projet, cette étude adopte une démarche essentiellement descriptive et il sera intéressant d'en compléter le contenu à travers deux nouvelles sources notamment afin d'étudier l'évolution de ce nouveau territoire touristique transfrontalier :

- des entretiens auprès des différents acteurs du projet afin de tester auprès d'eux les questions relatives à l'émergence d'un territoire touristique transfrontalier et à la gouvernance complexe liée à ce type de projet,

- les données chiffrées permettant d'évaluer définitivement les résultats des actions menées.

L'obtention de ces données permettra de répondre à la question de la prise de décision des randonneurs après la visite du site ou des médias sociaux de chacun des partenaires et l'un des enjeux futurs sera une mise en commun plus marquée des moyens et une attention plus précise portée à l'expérience vécue par les randonneurs sur les itinéraires de randonnée (Thanh Tan et Maingot, 2013).

\section{BIBLIOGRAPHIE}

N. ALEXANDRE-BOURHIS, S. KHALLA et C. ROUVRAIS CHARRON, « Quels métiers pour la communication numérique des organisations touristiques? ", Management et Avenir, 2013/2, nº 60, p. 195-211.

B. BARTIKOWKI, D. MERUNKA et P. VALETTE-FLORENCE, « L'attitude vis-à-vis des destinations touristiques : le rôle de la personnalité des villes ", Management et Avenir, 2008/4, n 18, p. 72-87.

S. BOLGHERINI et C. ROUX, « Les régions d'Europe et l'enjeu des coopérations », Pôle Sud, 2008/2, $n^{\circ} 29$, p. 111-130.

S. CARLISLE, M. KUNC, E. JONES et S. TIFFIN, « Supporting innovation for tourism development through multistakeholder approaches: Experiences from Africa », Tourism Management, 2013, vol. 35, p. 59-69.

P. CHHETRI, C. ARROWSMITH et M. JACKSON, « Determining hiking experiences in nature-based tourist destination », Tourism Management, 2004/1, vol. 25, p. 31-43.

F.-H. COURVOISIER et F.-A. COURVOISIER, « Communication et marketing d'institutions culturelles en suisse francophone », Management et Avenir, 2005/3, n 5, p. 133-150.

M. DEBABI et K. DAOUAS, «Les produits du terroir et écologiques comme vecteur d'innovation au tourisme responsable », Management et Avenir, 2015/2, n 76, p. 157-168.

O. DENERT et H. HUREL, « De l'espace frontalier au territoire transfrontalier », Labyrinthe, 2000/6 $\mathrm{n}^{\circ} 6$.

M. FILSER, « Le management des activités culturelles et de loisirs : questions stratégiques et état des recherches académiques », Management et Avenir, 2005/3, n 5, p. 179-189. 
H. FRANÇOIS, « Le tourisme durable une organisation du tourisme en milieu rural », Revue d'économie régionale et urbaine, 2004/1, p. 57-80.

C. GALlOUG et E. LEROUX, « E-tourisme, innovation et modes d'organisation », Management et Avenir, $2011 / 2, n^{\circ} 42$, p. 213-231.

N. HASCHAR-NOÉ, «L'aménagement des chemins de randonnée : un instrument d'identification et de gouvernance territoriales ", Espaces et sociétés, 2009/3, n 138, p. 115-133.

R. LADWEIN, «L'expérience de consommation, la mise en récit de soi et la construction identitaire : le cas du trekking ", Management et Avenir, 2005/3, n 5, p 105-118.

E. LEROUX, « Management du tourisme responsable, vecteur d'innovation environnementale, sociale, économique et territoriale ", Management et Avenir, 2015/2, n 76, p. 111-119.

C. MAUNIER, « Une approche triadique du marketing des destinations », Market Management, 2007/2, vol. 7, p. 41-64.

A. MARSAC, A.-M. LEBRUN et P. BOUCHET, « Tourisme durable et expériences touristiques : un dilemme. Proposition d'un dispositif d'analyse appliqué à l'itinérance en milieu rural », Management et Avenir, 2012/6, n 56, p. 134-153.

M. SAAYMAN et A. VILJOEN, « Who are wild enough to hike a wilderness trail? », Journal of Outdoor Recreation and Tourism, 2016, vol. 14, p. 41-51.

P. SAHUC, «Chemins périurbains : aménités vécues et enjeux réel », Natures Sciences Sociétés, 2010/2, vol. 18, p. 147-157.

M. SALVADOR-PERIGNON, « Tourisme culinaire et valorisation des produits artisanaux : vers un tourisme durable ", Management et Avenir, 2012/6, n 56, p. 114-133.

V. SIAU, « Sports de nature et attractivité des territoires », Pour, 2007/2, n 194, p. 13-20.

D.J. тімотну, « Cross-border partnership in tourism resource management: International parks along the US-Canada Border », Journal of Sustainable Tourism, 1999/7, n 3-4, p. 182-205.

V. THANH TAN et M. MAINGOT, «Comprendre les décisions d'achat dans les médias sociaux : le cas du e-tourisme ", Gestion, 2013/1, vol. 38, p. 30-38.

A. WEIDENFELD, « Tourism and cross border regional innovation systems », Annals of Tourism Research, vol. 42, 2013, p. 191-213.

\section{NOTES}

1. Source : https://fr.statista.com/statistiques/510847/classement-activites-sportives-prefereesfrance/

2. Source : Métropole européenne de Lille.

3. Source : Eurométropole Lille-Tournai-Courtrai. 


\section{RÉSUMÉS}

L'article traite d'une innovation-produit sur un territoire touristique transfrontalier émergent. Le programme Interreg IV a soutenu l'émergence d'un projet franco-belge de développement de la randonnée : «Inno Rando Pour Tous ». Ce projet a permis la mise en place d'une coopération transfrontalière avec la création de nouveaux produits touristiques et l'emploi d'une nouvelle application mobile. Les territoires concernés ont ainsi créé un produit à destination d'un public familial : «Rando Famili ». Après une présentation du cas d'étude, fondé sur un recueil qualitatif de données, nous proposons trois axes de réflexion liés à l'innovation-produit, à l'expérience de consommation et à l'émergence d'un nouveau territoire touristique.

This paper deals with an innovative product in a cross-border tourism destination. The Interreg IV program supported the emergence of the Franco-Belgian hiking development project "Inno Rando Pour Tous". This project enabled an original cross-border cooperation which led to the launch of new tourism products and the use of mobile techniques. The territories involved implemented a product addressing families, "Rando Famili". We will first present the case study based on a qualitative data collection, and then develop three perspectives related to product innovation, experiential marketing and the emergence of a new tourism destination.

\section{INDEX}

Mots-clés : innovation-produit, marketing touristique, territoire transfrontalier

Keywords : product innovation, tourism marketing, cross-border territory

\section{AUTEURS}

\section{FABRICE CAUDRON}

Maître de conférences, Université de Lille

E-mail : fcaudron@iaelille.fr

\section{CÉLINE THOMAS}

Ancienne responsable marketing, Intercommunale IDETA, Direction de la valorisation $\mathrm{du}$ territoire

E-mail : celthomas@yahoo.fr 